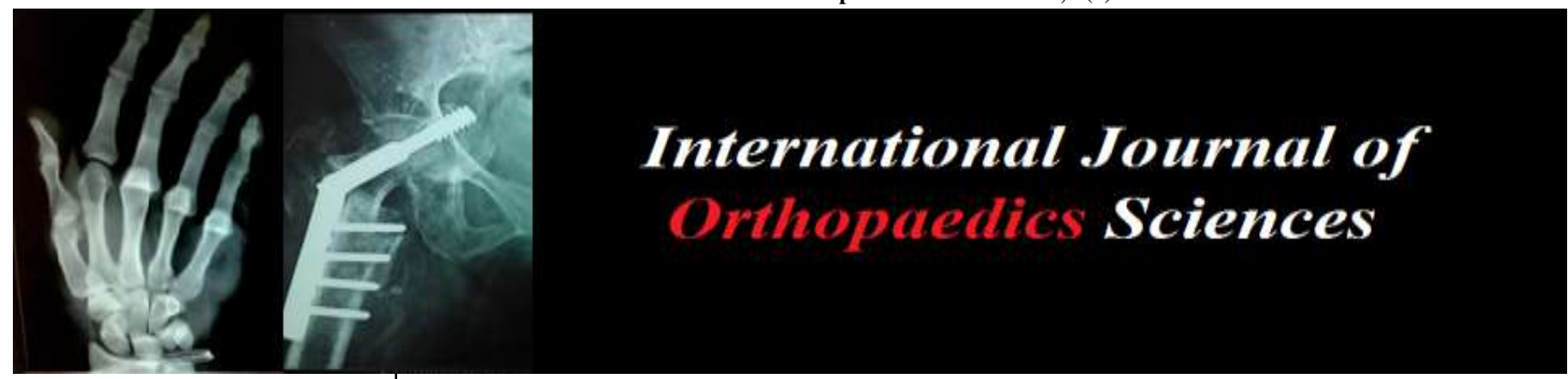

E-ISSN: 2395-1958

P-ISSN: 2706-6630

IJOS 2021; 7(4): 240-242

(C) 2021 IJOS

www.orthopaper.com

Received: 16-07-2021

Accepted: 25-08-2021

\section{Dr. BS Jayaram}

Kempegowda Institute of

Medical Sciences, Bangalore,

Karnataka, India

Dr. Lokesh M

Kempegowda Institute of Medical Sciences, Bangalore,

Karnataka, India

Dr. Karan CL

Kempegowda Institute of Medical Sciences, Bangalore,

Karnataka, India
Corresponding Author: Dr. Karan CL

Kempegowda Institute of Medical Sciences, Bangalore,

Karnataka, India

\section{Chronic achilles tendon rupture repair with plantaris tendon augmentation and anchor sutures: $A$ case report and review of literature}

\author{
Dr. BS Jayaram, Dr. Lokesh $M$ and Dr. Karan CL
}

DOI: https://doi.org/10.22271/ortho.2021.v7.i4d.2889

Abstract

The Achilles tendon is the strongest and largest tendon in the human body. A chronic rupture can be defined as a rupture with a delay in diagnosis or treatment for more than 6 weeks. It is a challenging problem. Reconstruction of the Achilles tendon depends on numerous factors such as the gap between the debrided tendon ends, the presence of a distal stump, and the presence of scar tissue. Various surgical techniques have been described. Open surgical options include augmentation with free fascia tendon graft, fascia advancement, local tendon transfer and synthetic or allograft augmentation. Here we report a case of a 55 year old male patient with a chronic degenerative tear in tendoachilles treated surgically with repair of the tendoachilles rupture with plantaris tendon augmentation with anchor sutures and its functional outcome.

Keywords: Achilles tendon, chronic tear, plantaris tendon

\section{Introduction}

The Achilles tendon is one of the most commonly ruptured tendons of the lower extremity. It commonly occurs in middle aged adults who are occasionally active. Chronic ruptures are more difficult to treat than acute injuries. Though chronic Achilles tendon rupture is not unusual, it is frequently misdiagnosed. According a study, about $20 \%$ of the Achilles tendon ruptures are neglected and diagnosed late ${ }^{[1]}$. About 1 week after rupture of Achilles tendon, any space between the ends gets filled with scar tissue. The patient usually feels a sharp snap with severe pain on the posterior part of his ankle with swelling. In patient with diabetes, pain may not be a predominant symptom due to neuropathy and hence may cause the patient to ignore the acute rupture event and continue walking on the affected leg, causing further damage ${ }^{[2]}$. The diagnosis is reliably made by a palpable tendon defect. Clinical tests have been described to test the continuity of the tendon which include Thomson's or Simmond's calf squeeze test, Matle's test, O'Brien's needle test.

There is no clear consensus on the optimal treatment of acute Achilles tendon rupture. Given the lack of prospective randomized trials and the small size of the studies, a standard procedure is lacking. Methods of reconstruction vary from direct repair, bridging of the tendon gap with tendon grafts or tendon transfers. Various operative procedures are described in literature which include augmentation with free fascia tendon graft, fascia advancement, local tendon transfer and synthetic or allograft augmentation. Here we report a case of a male patient with a chronic degenerative tear in tendoachilles treated surgically with repair of the tendoachilles rupture with plantaris tendon augmentation with anchor sutures and its functional outcome.

\section{Case report}

A 55-year-old male patient came to the out-patient department with a history of pain in the left ankle and difficulty walking since 3 months. The patient gave no history of trauma. On examination, the patient had tenderness over the left tendoachilles with inability to plantarflex left ankle. Thompson test was performed where calf muscle was squeezed with the patient lying in prone position and found to be positive with no plantar flexion of left ankle. Radiograph of left ankle was taken and no bony injuries were noted. 
Calcification was observed in the degenerated Achilles tendon. The patient was diagnosed to have a chronic rupture of left tendoachilles.

The patient was planned for surgical management with repair of left tendoachilles. With the patient in prone position, a 12 $\mathrm{cm}$ longitudinal incision was made medial to border of tendoachilles, and tendon sheath was opened in midline. Degenerative tear was noted and debridement was done and unhealthy torn ends were excised. With the foot held in plantar flexion the gap was found to be around $5 \mathrm{~cm}$ between the cut ends with a friable distal stump of the tendon. Plantaris tendon was found to be intact and was separated from its insertion on the calcaneus and dissected proximally and striped. Care should be given about sural nerve which lies in close proximity. The Plantaris tendon was passed through the proximal stump transversely and sutured to the distal stump and along the medial and lateral borders so as to form a dynamic loop. Good approximation was noted between the cut ends which improved on slight passive plantar flexion of ankle. The torn ends were sutured using anchor sutures by modified Kraków technique with the anchor fixed to the posterior aspect of calcaneum. The paratenon was closed and the wound was closed in layers. The ankle was immobilized in plantarflexion using a below knee plaster cast. Repair of the chronic tendoachilles rupture was done with plantaris tendon loop augmentation with anchor sutures and the operative time was 45 minutes. Sutures were removed on $12^{\text {th }}$ postoperative day.

The patient was followed up regularly and started on physiotherapy exercises at 6 weeks. At the end of 3 months, the patient was noted to be doing full weight-bearing mobilization with good active range of movements of the left ankle.

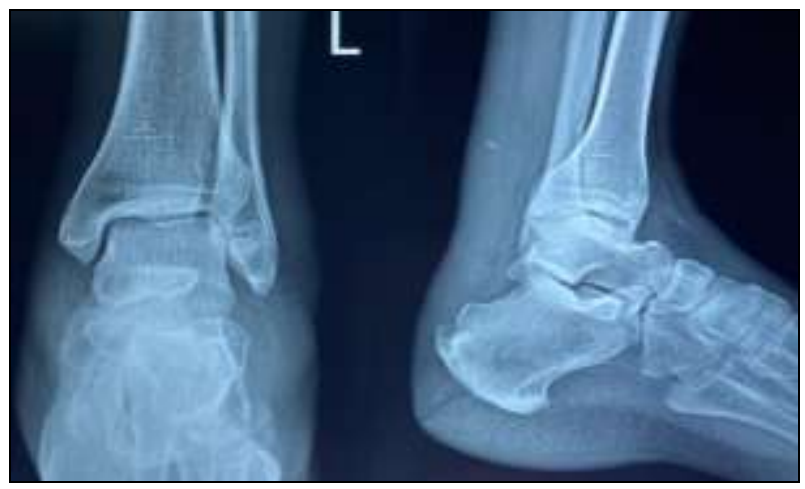

Fig 1: Pre-operative radiograph of left ankle showing no bony injuries were noted. Calcification can be seen in the degenerated Achilles tendon.

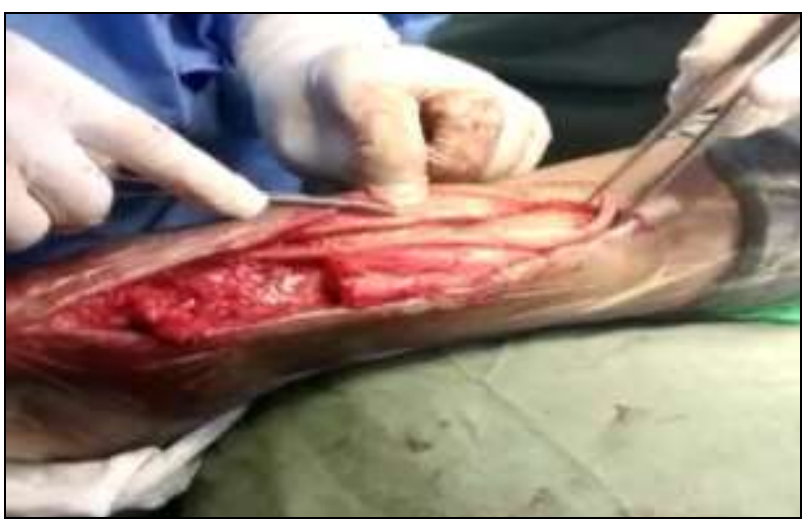

Fig 2: Plantaris tendon and sural nerve are identified and plantaris tendon is striped.

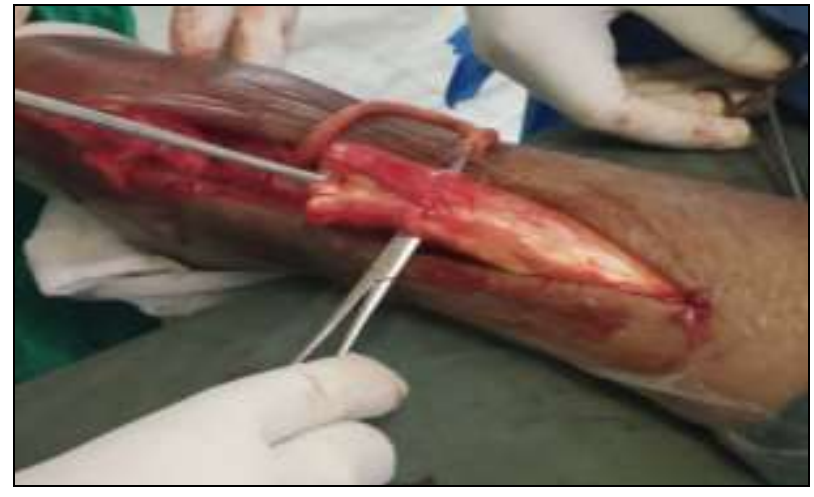

Fig 3: Plantaris tendon is made into a dynamic loop across the proximal stump.

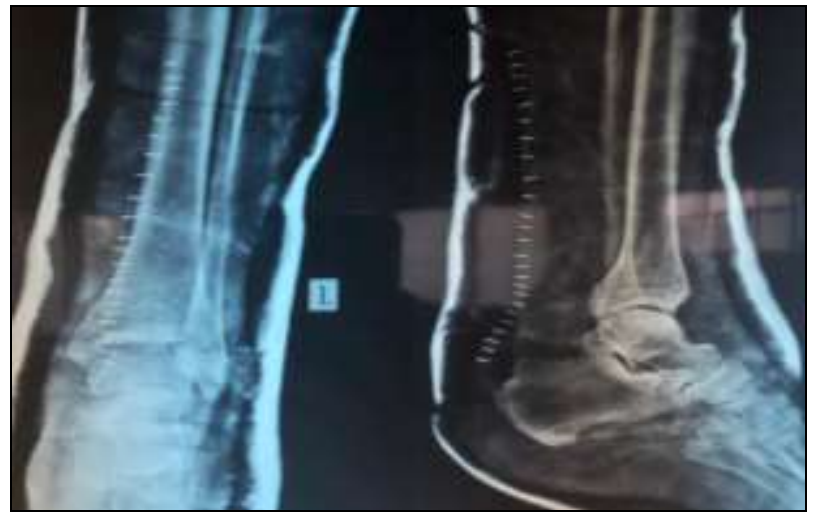

Fig 4: Post-operative radiograph of left ankle.

\section{Discussion}

The treatment of chronic Achilles tendon rupture presents a challenge to repair the large defect due to delay in appropriate treatment and to restore the function. The aim of surgical reconstruction is to restore full strength of the Achilles tendon and thus improve the activity level of patients ${ }^{[3]}$. The choice of surgical technique primarily depends on the time since injury, the length of the final gap between the two ends following debridement and the status of the tendon ends. If the tendon defect after debridement is less than $3 \mathrm{~cm}$ and less than 3 months has elapsed since the injury, direct repair is possible. However, a defect of more than $3 \mathrm{~cm}$ and if more than 3 months have passed since injury additional techniques such as local tendon transfer, tissue augmentation, synthetics and allografts can be used.

Various surgical procedures have been described for bridging the gap and restore the continuity of the tendon. Maffulli et al. [4] advocated the use of either peroneus brevis or flexor hallucis longus for reconstruction of chronic ruptures and indicated the need to carefully reinforce the attachment of the transferred tendon grafts to the stumps of the Achilles tendon to prevent pull-out. The Peroneus brevis tendon lies in close proximity to the achilles tendon but in a separate muscle compartment. It is in phase with the Gastrocnemius-soleus during normal gait cycle. Gallant et al. ${ }^{[5]}$ found mild objective eversion and plantar flexion weakness, but subjective assessment revealed no functional compromise following the procedure.

In a study on five patients with neglected tendoachilles rupture using a combination of peroneus brevis transfer and plantaris tendon augmentation found good functional results, low morbidity and satisfactory result for the patients. However, they described possibility of residual lateral ankle instability ${ }^{[6]}$.

Avadhanam Pavan Kumar et al. ${ }^{[7]}$ used a modification of the 
Bosworth technique in which a strip of the gastrocnemius aponeurosis was taken, made into a tendon-like structure and passed through the calcaneum after making a drill hole, then it was sutured back to the proximal stump. They found excellent results in sixty two patients and poor results in two patients. They reported delayed wound healing as one of the complications, however there were no re-ruptures. They described the technique to be ideally suited to zone I and II ruptures where end-to-end repair is not possible.

In a study done on four patients with chronic rupture described a method of repair which includes, V-Y gastrocnemius recession or advancement, excision of the fibroadipose defect, end-to-end anastomosis, plantaris tendon weaving to reinforce the anastomosis, and use of a pull-out wire suture. They found that all patients were satisfied with results and encountered no re-ruptures and returned to their preinjury activities ${ }^{[8]}$.

$\mathrm{K}$ L Wapner et al. ${ }^{[9]}$ described for reconstruction of chronic Achilles tendon rupture using the flexor hallucis longus tendon. Each patient developed a small but functionally insignificant loss in range of motion in the involved ankle and great toe. All patients had a satisfactory return of function.

In this case report we used a technique of repair with plantaris tendon augmentation with anchor sutures. Ethibond sutures can also be used in place of Anchor sutures. We found this technique to be simple and cost effective to treat tendoachilles tear with a gap between the cut ends less than $6 \mathrm{~cm}$. We did not encounter any problems related to wound healing. The patient is on regular follow-up and at the end of 6 months was found to have satisfactory results with good functional outcome.

\section{Conclusion}

A chronic tendoachilles rupture can occur due to delay in diagnosis or treatment. The diagnosis can be made based on clinical findings, but the management is technically more demanding than primary repair of acute rupture. Various operative procedures are described in the literature, but a standard procedure is lacking. We report a patient with a chronic degenerative tear in tendoachilles who was treated surgically with repair of the tendoachilles rupture with plantaris tendon augmentation with anchor sutures. We found this technique to be simple and cost effective with good functional outcome.

\section{References}

1. Ibrahim SA. Surgical treatment of chronic Achilles tendon rupture. J Foot Ankle Surg 2009;48(3):340-346. doi:10.1053/j.jfas.2009.02.007

2. Gabel S, Manoli A 2nd. Neglected rupture of the Achilles tendon. Foot Ankle Int 1994;15(9):512-517. doi:10.1177/107110079401500912

3. Ahmad J, Jones K, Raikin SM. Treatment of Chronic Achilles Tendon Ruptures With Large Defects. Foot Ankle Spec 2016;9(5):400-408. doi:10.1177/1938640016640895

4. Sebastian H, Datta B, Maffulli N, Neil M, Walsh WR. Mechanical properties of reconstructed achilles tendon with transfer of peroneus brevis or flexor hallucis longus tendon. J Foot Ankle Surg 2007;46(6):424-428. doi:10.1053/j.jfas.2007.07.003

5. Gallant GG, Massie C, Turco VJ. Assessment of eversion and plantar flexion strength after repair of Achilles tendon rupture using peroneus brevis tendon transfer. Am J Orthop (Belle Mead NJ) 1995;24(3):257-261.
6. Miskulin M, Miskulin A, Klobucar H, Kuvalja S. Neglected rupture of the Achilles tendon treated with peroneus brevis transfer: a functional assessment of 5 cases. J Foot Ankle Surg 2005;44(1):49-56. doi:10.1053/j.jfas.2004.11.003

7. Pavan Kumar A, Shashikiran R, Raghuram C. A novel modification of Bosworth's technique to repair zone I Achilles tendon ruptures. J Orthop Traumatol 2013;14(1):59-65. doi:10.1007/s10195-012-0222-y

8. Kissel CG, Blacklidge DK, Crowley DL. Repair of neglected Achilles tendon ruptures--procedure and functional results. J Foot Ankle Surg 1994;33(1):46-52.

9. Wapner KL, Pavlock GS, Hecht PJ, Naselli F, Walther R. Repair of chronic Achilles tendon rupture with flexor hallucis longus tendon transfer. Foot Ankle 1993;14(8):443-449. doi:10.1177/107110079301400803 